\title{
Enhancing somatic nuclear reprogramming by Oct4 gain-of-function in cloned mouse embryos
}

\author{
MARTIN J. PFEIFFER ${ }^{1}$, SEBASTIAN T. BALBACH ${ }^{1}$, TELMA C. ESTEVES ${ }^{1}$, \\ NICOLA CROSETTO ${ }^{2}$ and MICHELE BOIANI*,1 \\ ${ }^{1}$ Max-Planck Institute for Molecular Biomedicine, Münster and \\ ${ }^{2}$ Goethe University Hospital, Institute for Biochemistry II, Frankfurt am Main, Germany
}

\begin{abstract}
Cloned mouse embryo development to blastocyst stage correlates positively with the expression level of Oct4 (Pou5f1) at the morula stage, as reported previously by our laboratory. However, whether this correlation is based on a cause-effect relationship has remained unclear. To address this question, we artificially increased the level of Oct4 prior and subsequent to somatic cell nuclear transfer, by microinjection of Oct4 mRNA into ooplasts and by transgenic Oct4 induction at the morula stage, respectively. We observed higher developmental rates of cloned embryos to blastocyst when higher levels of Oct4 were superimposed with the initial reprogramming events; whereas increasing Oct4 at later stages of preimplantation development did not have a significant effect on developmental rates. Our results show that supplemental Oct4 facilitates oocyte-mediated reprogramming only during the first cleavages, implying that the higher Oct4 level observed in developmentally competent cloned morulae is a readout of reprogramming events that successfully took place earlier.
\end{abstract}

KEY WORDS: Oct4, Pou5f1, reprogramming, stem cell, somatic cell nuclear transfer, early development

\section{Introduction}

The prospect of efficiently creating pluripotent cells from adult tissues via nuclear or cellular reprogramming - the experimental conversion of a differentiated cell state to a less differentiated or pluripotent state - is appealing to stem cell research, disease modeling and regenerative medicine. The mechanisms of reprogramming may vary according to the experimental platform used, as suggested by the different rates and extents of reprogramming achieved. To date, the oocyte is the only known cell of the adult body endowed with native reprogramming ability, and reprogramming induced by the oocyte after somatic cell nuclear transfer (SCNT) is the only one that affords totipotency. SCNT and fusion of somatic cells with pluripotent partners share the ability to reprogram a somatic cell nucleus within 48 hours and, to a certain extent, independently of cell divisions (Bhutani et al., 2010; Han et al., 2008). In contrast to SCNT and cell fusion, the generation of induced pluripotent stem (iPS) cells by over-expression of Oct4, Sox2, Klf4 and Myc takes about two weeks, is highly dependent on cell divisions and remains very inefficient (Takahashi et al., 2006; Zhao et al., 2008; Hanna et al., 2009). Notwithstanding distinctions, all reprogramming platforms seem to share common reprogramming agents (Hochedlinger et al., 2009; Egli et al., 2010), although their importance and impact across different settings has not been thoroughly assessed. Among the candidate agents, the transcription factor Oct4 is the most popular, albeit not the only common factor involved.

Oct4 has an essential role in pluripotency and development: it is not possible to derive embryonic stem (ES) cells from the inner cell mass (ICM) of Oct4-1- mouse blastocysts (obtained from the intercross of $\mathrm{Oct4}^{+/-}$parents) (Nichols et al., 1998). Tsuji and colleagues reported a similar phenotype when they delivered anti-Oct4 morpholino to 2-cell stage mouse embryos (Tsuji et al., 2006). Furthermore, morpholino mediated knock-down of OCT4 in mouse zygotes results in an early and dose-dependent cleavage arrest (Foygel et al., 2008).

Cloned mouse embryos express lower levels of Oct4 than fertilized counterparts and, in line with the known effects of Oct4

Abbreviations used in this paper: ES, embryonic stem cell; ICM, inner cell mass; ICSI, intracytoplasmic sperm injection; iPS, induced pluripotent stem cell; luc, luciferase; Oct4 (Pou5f1), POU domain class 5 transcription factor 1; SCNT, somatic cell nuclear transfer; TE, trophectoderm.

\footnotetext{
*Address correspondence to: Michele Boiani. Max-Planck Institute for Molecular Biomedicine, Röntgenstrasse 20, D-48149 Münster, Germany. Fax: +49-251-70365-399. e-mail: mboiani@mpi-muenster.mpg.de
} 
depletion in pre-implantation, also exhibit a reduced developmental fitness. In particular, development of cloned mouse embryos beyond the morula stage correlates positively with Oct4 levels in the morula itself (Cavaleri et al., 2008). Whether this correlation indicates a cause-effect relationship has not been determined to date.

In this study we aimed to determine whether Oct4 is a driving force or merely a marker during mouse oocyte-mediated reprogramming and cloned embryo development, by using gain-offunction methodology. Oocytes have a built-in reprogramming machinery, whose components can be dissected and evaluated for importance by the use of micromanipulation techniques and transgenic approaches. To test if higher levels of Oct4 improve reprogramming, we used two experimental settings: 1) microinjection of Oct4 mRNA into the mouse oocyte prior to SCNT, and 2) Oct4 induction after SCNT from donor cells that carry an inducible Oct4 transgene (Fig. 1). We analyzed the effects of these perturbations on reprogramming efficiency and cloned embryo development. Injection of mRNA in the ooplasm prior to SCNT leads to a defined increase of Oct4 message and protein during the first cell cycles, when the initial and most extensive reprogramming events are considered to take place. The transgenic approach allows for Oct4 increase at later cleavage stages, when microinjection techniques are cumbersome to perform on all blastomeres of the embryo. Using this dual approach we dissected the role of Oct4 levels in oocyte-mediated reprogramming at different developmental stages.

Our data demonstrate a beneficial effect of Oct4 during the initial but not the later cleavage stages of cloned mouse embryo development. This discrepancy strongly suggests that Oct4 pro- motes or facilitates the initial steps of mouse oocyte-mediated reprogramming, whereas at later preimplantation stages, the observed positive correlation between the level of Oct4 and further development is a consequence of earlier reprogramming events.

\section{Results}

\section{Increasing Oct4 level by mRNA microinjection does not benefit normal mouse development}

In order to increase Oct4 levels in the mouse ooplasm prior to SCNT, we first developed a microinjection protocol in mouse zygotes using in vitro transcribed mRNA. In vitro synthesized mRNA encoding N-terminal FLAG-tagged Oct4 was co-injected with red fluorescent dextran beads as a tracer to ensure that all embryos received similar injection volumes. In this setting, the oocyte poses an upper limit to the volume that can be injected; therefore the amount of Oct 4 mRNA supplied depends on the concentration, not on the volume. As a control we injected zygotes with corresponding concentrations of mRNA encoding luciferase (LUC) to account for the physical effects of micromanipulation and mRNA injection. The tracer does not seem to interfere with normal development as the embryos injected with dextran beads developed to term at substantial rates after embryo transfer (data not shown).

We verified the increase of the intracellular Oct4 mRNA after injection of in vitro synthesized mRNA. To this end we applied qRT-PCR using a combination of primers that were designed to discern between endogenous and injected Oct4 mRNA. As expected, injection of increasing concentrations of mRNA resulted

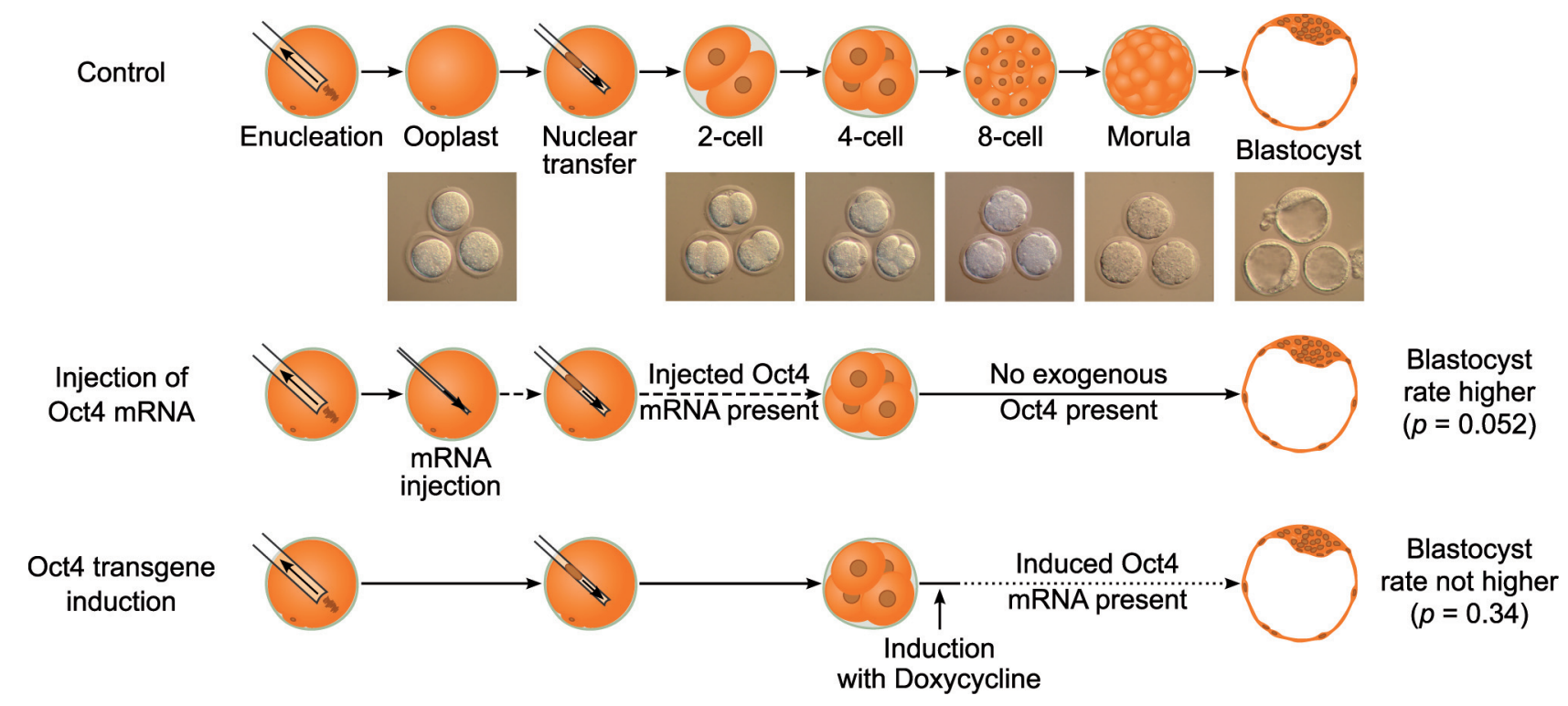

Fig. 1. Scheme of the experimental design used in this study. To assess the impact of Oct4 elevation on oocyte mediated reprogramming, two independent series of somatic cell nuclear transfer (SCNT) experiments were performed. On the one hand, Oct4 mRNA was microinjected into enucleated oocytes prior to the tranplanstation of a somatic nucleus followed by assessment of subsequent preimplantation development. In this setting, Oct4 is elevated only throughout the first cell divisions and the treatment lead to higher developmental rates of the cloned embryos. On the other hand, to assess the impact of Oct4 on late reprogramming events, a transgenic Oct4 inducible system was used. Oct4 was induced by adding doxycyline to the medium during the 4- to 8-cell transition, leading to transgenic Oct4 expression only during late reprogramming events. In this experiment, no significant change in developmental rates to blastocyst was observed in induced versus un-induced controls. 
in an increase of the intracellular amount of FLAG-tagged Oct4coding mRNA (Fig. 2A). Total Oct4 mRNA also increased after microinjection 2-, 7-, 15- or 48-fold after the injection of 1, 5, 10 or $50 \mathrm{ng} / \mu \mathrm{l}$ mRNA solution, respectively, when compared to an uninjected control.

As the injection of high concentrations of Oct4 mRNA into mouse zygotes has been reported to impair development (Foygel et al., 2008), we first determined the mRNA concentration range that could be injected into zygotes without harming normal development. In comparison to luc mRNA-injected control embryos, Oct4 mRNA concentrations above $10 \mathrm{ng} / \mu \mathrm{l}$ injected at the 1 cell stage significantly decreased the developmental rate in a dosedependent manner (Fig. 2C). Altogether our results confirm the previous finding that too much Oct4 impairs early mouse embryonic development, emphasizing the importance of a tight regulation of Oct4 in the initial stages of development. In this context, it may be noted that perturbations of Oct4 levels as little as 2-fold lead to differentiation of pluripotent mouse ES cells (Niwa et al., 2000).

Since Oct4 is known to drive its own promoter in ES cells (Boyer et al., 2006), we next examined the possible influence of microinjected Oct4 mRNA on the amount of Oct4 transcribed from the nuclear locus. qRT-PCR conducted at the 2-cell stage revealed a reduction of endogenous Oct4 in a dose dependent manner (Fig. 2B). These findings may be explained by a mechanism dampening fluctuations of Oct4 level in early embryos, for instance via increased rate of maternal mRNA degradation. In fact, knockdown of OCT4 during the maternal-to-embryonic transition was shown to interfere with maternal mRNA degradation (Foygel et al., 2008).

In summary, we established that the microinjection of mouse zygotes with Oct $4 \mathrm{mRNA}$ at $1 \mathrm{ng} / \mu \mathrm{l}$ increases total Oct $4 \mathrm{mRNA}$ amount without affecting subsequent development. This nontoxic concentration was subsequently applied to ooplasts prior to SCNT in order to test the effects of Oct4 on the efficiency of reprogramming in cloned mouse embryos.

\section{SCNT embryos benefit from additional Oct4 during early reprogramming events}

The greatest part of reprogramming is considered to take place during the first two cell cycles after nuclear transfer. In order to determine the consequences of imposed higher level of Oct4 on oocyte-induced reprogramming, we injected FLAG-Oct4 coding mRNA (1 ng/ $\mu \mathrm{l})$ into enucleated oocytes (i.e. ooplasts) prior to SCNT from ovarian cumulus cells (Fig. 1). With this procedure, the amount of total Oct4 message could be doubled transiently (Fig. 2A), allowing us to assess the impact of Oct4 increase on initial reprogramming events. It should be noted that mRNA microinjection entails an additional micromanipulation step in comparison to the conventional cloning procedure (enucleation, SCNT) and that each micromanipulation step is potentially harmful to the embryo; therefore control ooplasts were microinjected with luc mRNA to account for the effects of the additional micromanipulation. In three independent experiments using the same mRNA stocks, we monitored development of cloned embryos derived from ooplasts injected with $1 \mathrm{ng} / \mu \mathrm{l}$ Oct4 mRNA prior to SCNT, and compared them to luciferase-injected controls. We observed higher developmental rates of cloned embryos derived from ooplasts injected with FLAG-Oct4 coding mRNA (22\%
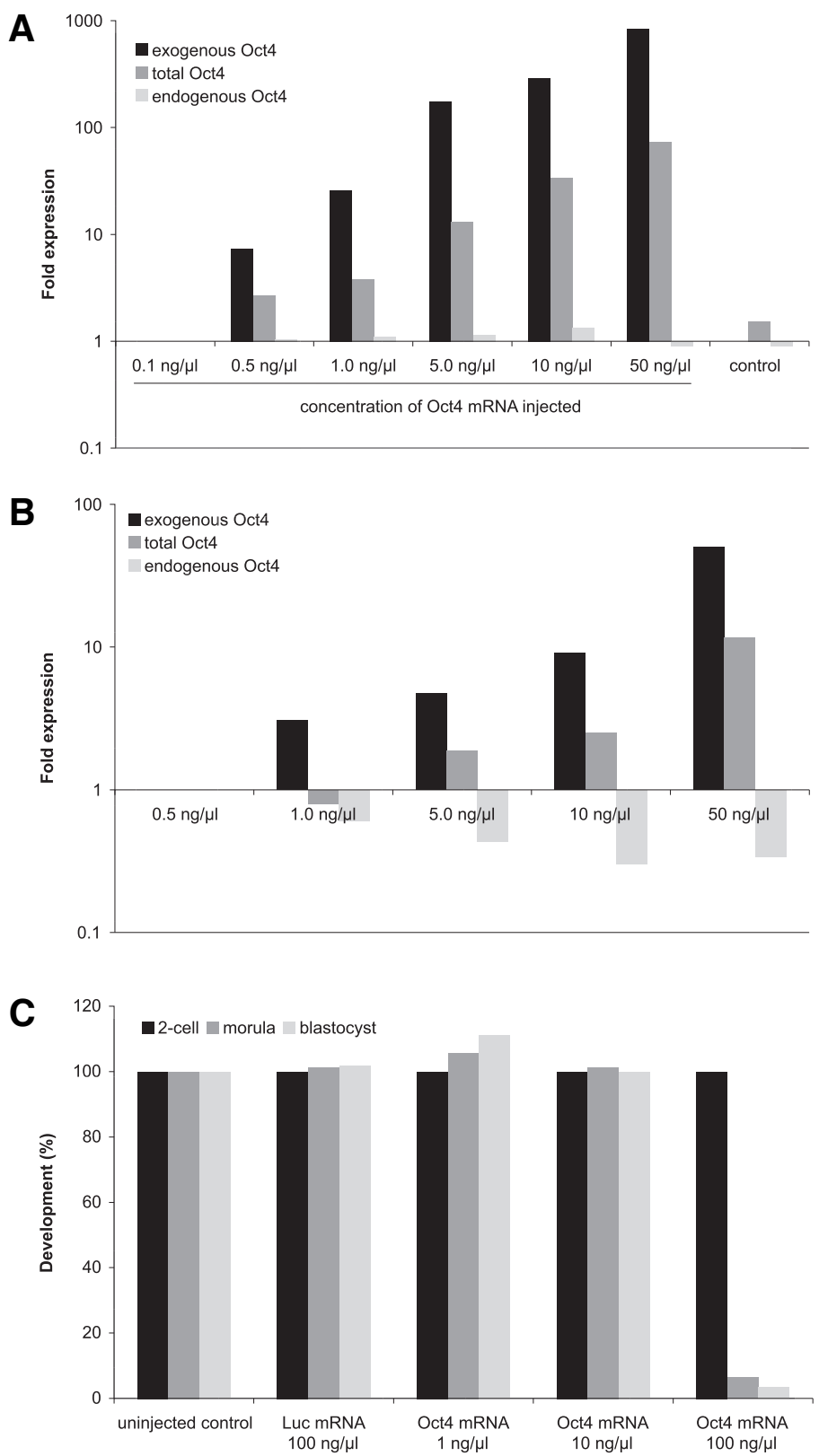

Fig. 2. Effect of mRNA injection in fertilized mouse pre-implantation embryos. (A) qRT-PCR data on pools of five oocytes injected with increasing concentrations of FLAG-Oct4 mRNA. Data is normalized to oocytes injected with a concentration of $0.1 \mathrm{ng} / \mu /$ (B) qRT-PCR data on pools of five 2-cell embryos injected with increasing concentrations of FLAG-Oct4 mRNA at the zygote stage. The data is normalized to embryos injected with a concentration of $0.5 \mathrm{ng} / \mu \mathrm{l}$. (C) Developmental rates of embryos injected at the zygote stage with FLAG-Oct4 or luciferase mRNA.

developed to blastocyst, versus $14 \%$ for embryos derived from luc mRNA-injected counterparts; Fisher's test, $p=0.052)$. This difference was already detectable at the 4 -cell stage (63\% of the FLAGOct4 mRNA-injected ooplasts developed to 4-cell, versus $57 \%$ for embryos derived from luc mRNA injected counterparts; Fisher's test, $p=0.26$; Table 1). To explore the cellular foundations of 
improved reprogramming after Oct4 mRNA injection, we analyzed cell lineage markers in cloned blastocysts developed from ooplasts microinjected with FLAG-Oct4 mRNA and compared them to cloned control embryos. qRT-PCR analysis of Oct4, as well as triple immunostaining of the pluripotency markers OCT4 and NANOG and the trophectoderm (TE) marker CDX2, detected no differences between the two groups (Fig. 3). Notably, OCT4 was immunostained in the TE, which is not unusual for early blastocyst stage mouse embryos (Boiani et al., 2002; Balbach et al., 2010) and has also been reported for bovine blastocysts (Kurosaka et al., 2004). Clearly, the OCT4 protein can persist the silencing of its gene in the TE and can still be detected when the mRNA is already starting to become ICM-restricted. Taken together, these results suggest that indeed, SCNT derived embryos benefit from additional Oct4 during early reprogramming events, which positions Oct4 as an important member of the oocyte's reprogramming machinery.

Given the importance of Oct4 during oocyte-induced reprogramming, we analyzed Oct4 transcript levels in single oocytes via qRT-PCR at the time when they would be subjected to SCNT. We observed high heterogeneity, namely: a variation of more than one order of magnitude across the individual oocytes analysed (Fig. 4). Gene expression data from minute specimens, such as individual oocytes, may be influenced by inadvertent slight differences in sample processing even if a housekeeping gene transcript (i.e. Gapdh) is included. Since our results show that oocytes microinjected with Oct4 mRNA support higher cloning rates ( $14 \%->22 \%$ blastocyst formation), we envision that the heterogeneous Oct4 content of unmanipulated oocytes may

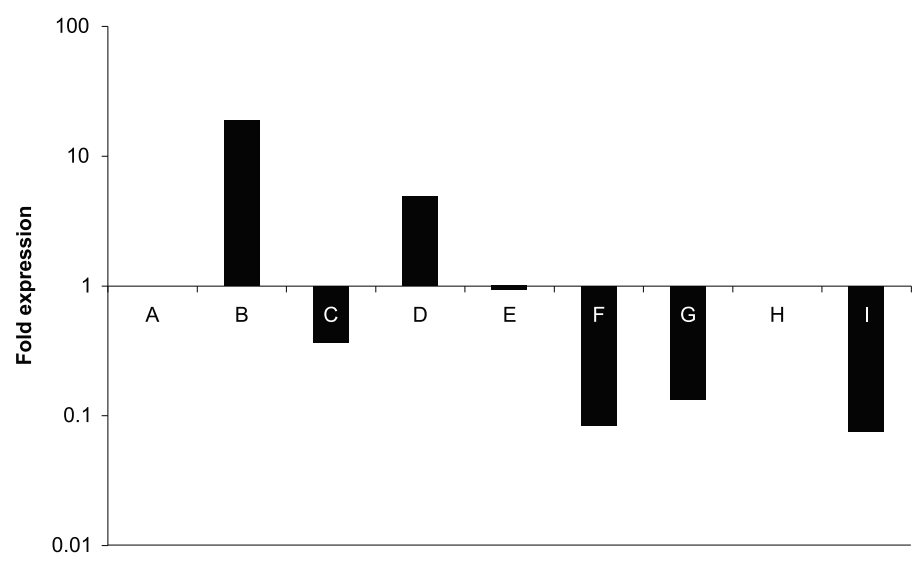

Fig. 4. Heterogeneity of Oct4 expression among individual mouse oocytes. Oct4 expression measured in single oocytes. Data normalized first to Gapdh expression and then to one oocyte chosen randomly. A large variation between individual oocytes can be observed.
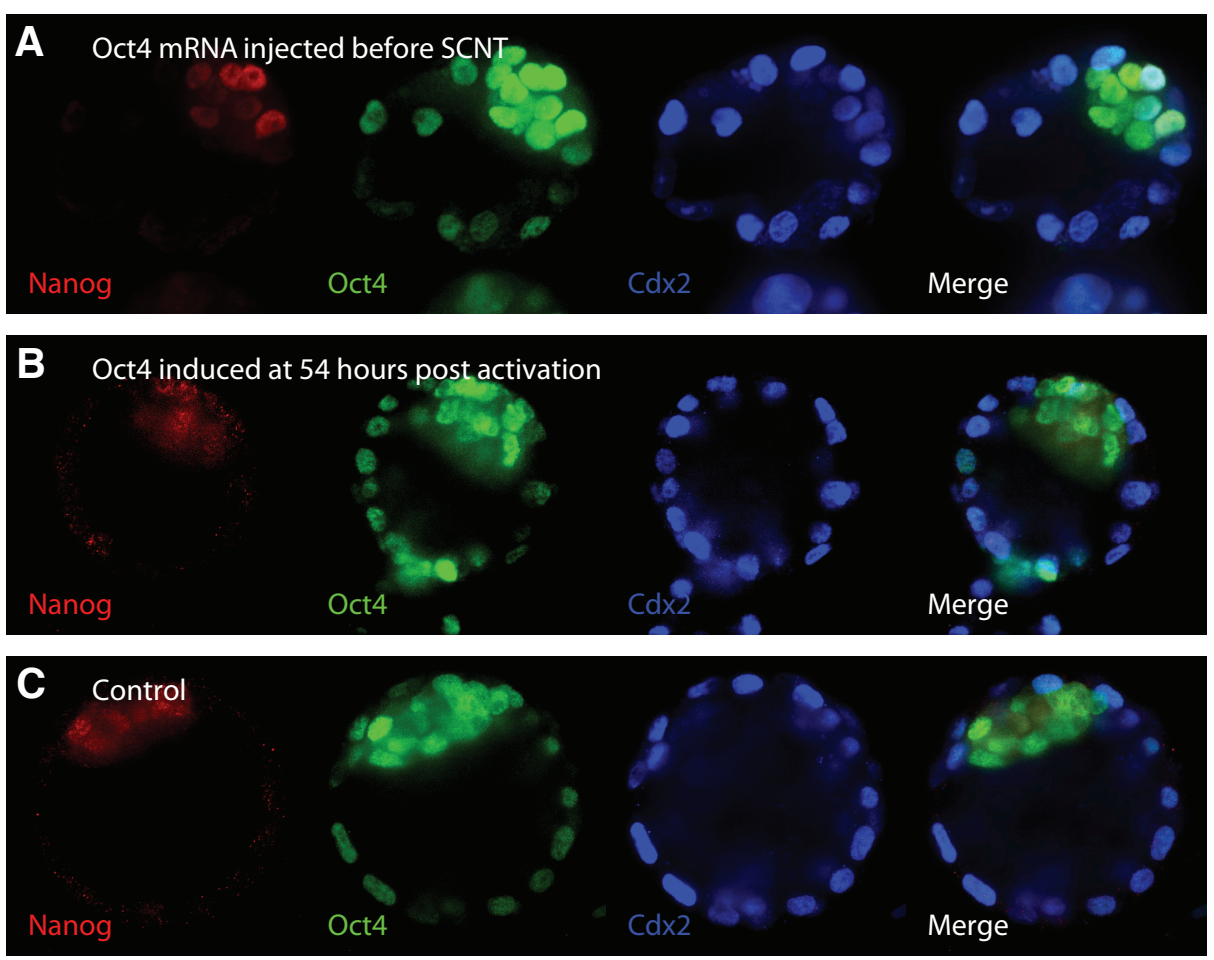

Fig. 3. Immunohistochemistry for early developmental markers in cloned embryos. Blastotrophectoderm is typically found in SCNT embryos (A) SCNT into ooplasts injected with $1 \mathrm{ng} / \mathrm{\mu l}$ FLAG-Oct4 mRNA (B) SCNT of a OG $2 \times$ tetOct4 cumulus cell with induction of the Oct4 transgene (C) SCNT control embryo.

account, at least in part, for the heterogeneous reprogramming abilities and variable cloning rates observed after conventional SCNT.

\section{Impact of Oct4 on late reprogramming events}

Cavaleri and colleagues reported that cloned embryos with higher levels of Oct4 at the morula stage have higher developmental success (Cavaleri et al., 2008). However, it is not clear whether Oct4 is the cause for success, or merely reflects successful reprogramming. To address this question, we increased Oct4 mRNA amount in the morula instead of the oocyte. The described microinjection protocol allows manipulation of mRNA only at early stages, because microinjection of Oct4 mRNA becomes highly impractical (if not unfeasible) in cleavage-stage embryos beyond the 4-cell stage. Therefore, nuclei from a mouse strain carrying an Oct4 transgene under the control of a tetracycline responsive element (TRE) were used for nuclear transfer experiments. This

TABLE 1

DEVELOPMENTAL RATES OF CLONED MOUSE EMBRYOS

\begin{tabular}{lccccc} 
& 1-cell & 2-cell & 4-cell & morula & blastocyst \\
\hline Oct4 & $100(202)$ & $86(173)$ & $63(127)$ & $42(85)$ & $22(45)$ \\
Luc & $100(195)$ & $83(161)$ & $57(111)$ & $35(68)$ & $14(28)$ \\
\hline
\end{tabular}

Enucleated oocytes where injected with $1 \mathrm{ng} / \mu \mathrm{l}$ FLAG-Oct4 mRNA or $1 \mathrm{ng} / \mu \mathrm{l}$ luciferase (luc) mRNA prior to transfer of cumulus cell nuclei. Values are percentage of oocytes manipulated for SCNT (1-cell), and total number of embryos (in brackets). 
A

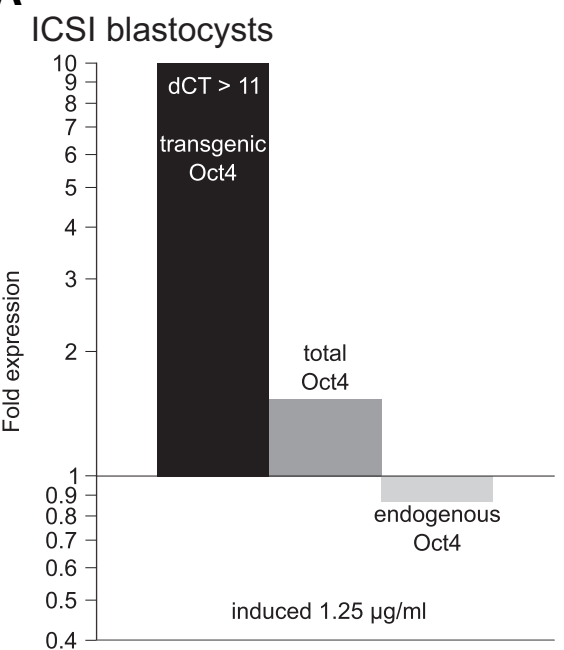

B

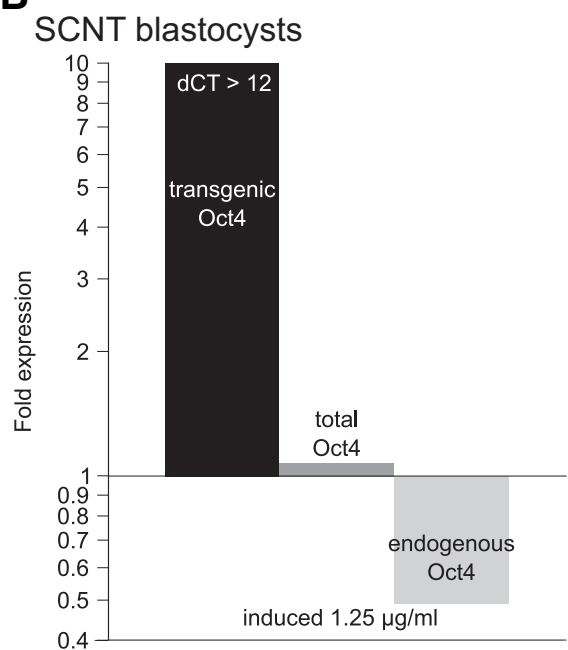

Fig. 5. Increase of Oct4 mRNA levels in ICSI and SCNT late pre-implantation embryos. (A) Oct4 expression in embryos derived from intracytoplasmic injection of sperm from a tetOct4 donor (ICSI), in the presence (induced) of $1.25 \mu \mathrm{g} / \mathrm{ml}$ doxycycline. (B) Oct4 expression in embryos derived from SCNT of a OG2 x tetOct4 cumulus cell into a B6C3F1 ooplast in the presence (induced; 54 hours post-activation) of $1.25 \mu \mathrm{g} / \mathrm{ml}$ doxycycline. All data is normalized to un-induced control embryos.

transgenic system allows Oct4 induction upon addition of doxycycline to the culture medium (Hochedlinger et al., 2005; Fig. 1).

Donor mice were generated by intercross of homozygous tetOct4 male mice and homozygous OG2 female mice, to match the genetic background of mice used in previous experiments (Cavaleri et al., 2008). To test the transgene induction parameters, embryos derived by intracytoplasmic sperm injection (ICSI) of tetOct4 sperm into OG2 oocytes were cultured in the absence or presence of $1.25 \mu \mathrm{g} / \mathrm{ml}$ doxycycline in the medium. qRT-PCR analyses of resultant blastocysts revealed a clear induction of the transgene (Fig. 5). Interestingly, the total amount of Oct4 transcript was only slightly increased in induced compared to uninduced embryos. Endogenous Oct4 transcript levels were slightly reduced in induced embryos, indicating a possible negative regulatory feedback. In line with the transcript data, no differences between induced and un-induced embryos could be observed at the protein level as measured by immunocytochemistry (Fig. 3).

We then performed two independent nuclear transfer experiments using ovarian cumulus cells of tetOct4-OG2 mice. After activation, these cloned embryos were cultured in presence or absence of $1.25 \mu \mathrm{g} / \mathrm{ml}$ doxycycline, and their developmental rates

TABLE 2

\section{DEVELOPMENTAL RATES OF CLONED MOUSE EMBRYOS DERIVED WITH OG2 $X$ tetOCT4 AS CUMULUS CELL NUCLEAR DONORS}

\begin{tabular}{lccccc} 
& 1-cell & 2-cell & 4-cell & morula & blastocyst \\
\hline Oct4 induced & $100(119)$ & $92(109)$ & $83(99)$ & $78(93)$ & $39(46)$ \\
un-induced & $100(117)$ & $91(106)$ & $83(97)$ & $74(86)$ & $32(38)$ \\
\hline
\end{tabular}

The transgenic Oct 4 was induced transferring the embryos to $1.25 \mu \mathrm{g} / \mathrm{ml}$ doxycycline containing media prior to the morula stage (54 hours post activation). Values are percentage of oocytes manipulated for SCNT (1-cell), and total number of embryos (in brackets).

where compared. We observed higher developmental rates of transgenic SCNT embryos to 4-cell compared to regular (nontransgenic) B6C3F1 cloned embryos. However, these differences were no longer detectable at the blastocyst stage. Interestingly, embryos that were first cultured in normal medium and then transferred to doxycyline-containing media at the 4- to 8cell stage ( 54 hours post activation) showed similar developmental rates compared to un-induced embryos. Specifically, developmental rates to blastocyst were $39 \%$ and $32 \%$, for Oct4-induced (at 54 hours) and un-induced embryos, respectively (Fisher's test, $p=0.34$ ). Furthermore, we measured Oct4 promoter driven GFP levels at the morula stage and did not detect differences between the doxycyline-treated compared to the untreated group (Fig. 6). It is formally possible that fluorescence levels were not in the linear range of detection, whereby lack of a measurable difference is just a technical artifact. However, independent of the treatment, the probability of a morula stage embryo to form a blastocyst clearly correlated with GFP intensities, as previously reported by our laboratory (logistic regression model, t distribution, $p=9.06 \cdot 10^{-5}$; Cavaleri et al., 2008).

qRT-PCR for Oct4 showed an induction of transgenic Oct4 in doxycycline-treated inducible cloned blastocysts. However, the total amount of Oct4 was only slightly increased (Fig. 5). In contrast, no transgenic Oct4 mRNA could be detected in doxycycline-treated 2-cell embryos (data not shown). In agreement with the qRT-PCR data, immunostaining of OCT4, NANOG and CDX2

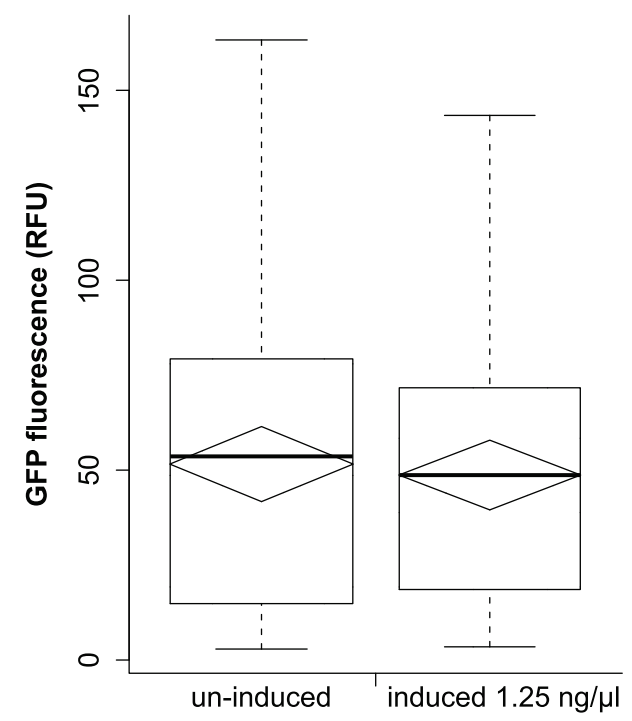

Fig. 6. Distribution of fluorescence intensity measured at the morula stage, for doxycycline-induced (54 hours post activation) or uninduced SCNT embryos. Box plot: top and bottom lines indicate interquartile range, middle line indicates median; whiskers indicate range of variation limited to 1.5 times inter-quartile range. 
in SCNT-derived blastocysts did not reveal differences between the different treatment groups (Fig. 3).

\section{Discussion}

In this study, we have shown that cloned mouse embryos benefit from increased Oct4 level imposed in the ooplasm prior to SCNT from ovarian cumulus cells. However, increasing Oct4 levels in cloned embryos at the 4- to 8-cell stage did not benefit developmental rates significantly. Taken together, these findings indicate an involvement of Oct4 in early reprogramming events in a SCNT setting and shed light on the molecular foundation for the reported correlation between Oct4 levels at the morula stage and developmental success of SCNT embryos (Cavaleri et al., 2008). Importantly, our findings portray the late reprogramming events as Oct4-independent. Therefore, when examined at the morula stage, Oct4 expression is not driving the correlation observed between Oct4 levels and developmental competence of cloned embryos, but rather, it is a readout of successful reprogramming events accomplished earlier.

\section{Role of Oct4 during the first cleavages}

As observed after intercross of $\mathrm{Oct}^{+/-}$mice, Oct4 ${ }^{-1-}$ embryos are able to develop to blastocyst, but fail to establish a pluripotent ICM (Nichols et al., 1998). In these experiments, the Oct4-1zygote still harbored maternal Oct4 transcript and protein; therefore it can be hypothesized that possible effects of Oct4 on early events (e.g. during cleavage) were masked by the presence of maternal Oct4, and that maternal Oct4 is required for the survival of $\mathrm{Oct4}^{-1-}$ embryos through initial cleavages. Indeed, blocking translation of Oct4 mRNA by injection of a specific anti-sense morpholino led to a developmental arrest at the one- to multi-cell transition (Foygel et al., 2008). Interestingly, also increasing Oct4 by injecting high concentrations of Oct4 mRNA into zygotes results in developmental failure, as shown in the present study and in a previous report (Foygel et al., 2008). Specifically, when injected at very high concentrations, the treated embryos are not able to develop beyond the 2-cell stage (Fig. $2 \mathrm{C}$ ). It is conceivable that a massive increase of Oct4 leads to an unphysiological occupancy of OCT4 binding sites, which are prevented from establishing their normal molecular interactions. The OCT4 interactome is large and includes many proteins with crucial functions during the earliest events of development (Pardo et al., 2010; van den Berg et al., 2010); amongst those are members of the NuRD complex and SMARCA4 (BRG1), whose depletion in mouse embryos has been reported to cause developmental arrest at the 2- to 4-cell stage (Bultman et al., 2000) and whose overexpression facilitates reprogramming in the iPS setting (Singhal et al., 2010). Corroborating this hypothesis is the recent finding that ooplasts only retain their developmental competence after SCNT when a sufficient amount of nuclear factors like SMARCA4 are maintained after enucleation (Egli et al., 2010).

It has been suggested that Oct4 facilitates zygotic genome activation and enhances maternal mRNA degradation (Foygel et al., 2008). In our experiments, we observed less endogenous Oct4 transcript at the 2-cell stage after injection of in vitro synthesized Oct4 mRNA into zygotes. This result supports the hypothesis that Oct4 influences the degradation of maternal mRNA at the maternal-to-embryo transition. An alternative expla- nation could be a feedback loop, in place to stabilize total Oct4 mRNA levels (homeostasis). This possibility is supported by the observation that morpholino-mediated knockdown of OCT4 results in an overexpression of endogenous Oct4 (Foygel et al., 2008). It is interesting to note that Oct4 is expressed in germ cells and upregulated during oocyte maturation (Pesce et al., 1998). However, the mature oocyte contains relatively little OCT4 protein as compared to later stages of pre-implantation development (Tang et al., 2009; Liu et al., 2004). Our qRT-PCR data from single oocytes further indicate that the amount of Oct4 transcript, while generally low, is highly variable amongst individual oocytes (Monti et al., 2009). This may partially explain the high variability existing among oocytes in their ability to support reprogramming and cloned embryo development after SCNT.

\section{Role of Oct4 in reprogramming}

Until recently, no experimental reprogramming platform had been able to revert a somatic cell back to a pluripotent state without overexpression of Oct4 (Takahashi et al., 2006; Feng et al., 2009; Kim et al., 2009). However, Heng and colleagues reported that the transcription factor $\mathrm{Nr5a2}$, which is also present in the oocyte, is able to replace Oct4 in the iPS technology (Heng et al., 2010), suggesting that Oct4 is dispensable for reprogramming. Furthermore, little is known about the molecular mechanisms of reprogramming. In this context, different platforms have different limitations. Even though reprogramming using the SCNT approach is efficient and fast, obtaining sufficient amounts of biological material for common experimental methods is painstaking and time-consuming. On the other hand, while it is relatively easy to transduce millions of cells simultaneously to generate iPS cells, the inefficiency of the reprogramming process leads to large heterogeneity in the cell population, which in turn makes identification and analysis of early molecular events during reprogramming extremely complicated. Despite these limitations, certain considerations about the mechanisms governing reprogramming in different platforms can be made. In the case of iPS cells, it has been shown that the stoichiometry of reprogramming factors, and not just their absolute levels, influence rates of iPS cell colony formation (Papapetrou et al., 2009). During reprogramming, a wealth of epigenetic changes has to be introduced, among them de-methylation of promoters and extensive modifications of histone marks. As somatic cells are not capable of active DNA de-methylation, reprogramming in the iPS setting is highly dependent on gradual activation of necessary promoters during cell divisions. Binding of pluripotency-related transcription factors to these transiently hemi-methylated gene promoters may lead to passive loss of DNA methylation because they prevent access of DNA methyltransferases to these sites. Therefore, reprogramming in the iPS setting can be made more efficient by increasing the rate of cell division, through treatment with chemicals that cause wider fluctuations of gene expression, and by introducing transcription factors that are able to stabilize these fluctuations into states compatible with pluripotency (Feng et al., 2009).

While sensible in an iPS cell scenario, these mechanistic considerations may not apply to oocyte-mediated reprogramming. The oocyte is capable of active DNA de-methylation and is rich in chromatin remodeling complexes designed to act upon the paternal genome after fertilization. As a result, reprogramming 
factors necessary or supportive in an iPS scenario may not at all be required in the reprogramming machinery of the oocyte. It is conceivable, however, that increasing certain factors improves the reprogramming capacity of oocytes. Based on this consideration, we injected Oct4 mRNA into mouse ooplasts prior to nuclear transplantation. The injected concentration $(1 \mathrm{ng} / \mu \mathrm{l})$ led to a 2-fold increase in total Oct4 transcript in pooled oocytes. This increase was transient and disappeared after 24 to 48 hours. However, even though the externally supplied Oct4 was present for only a short period of time, it increased the developmental potential of cloned mouse embryos, arguing for superior reprogramming directly linked to the increase in Oct4. As OCT4 is known to interact with chromatin remodeling complexes (Pardo et al., 2010; van den Berg et al., 2010), it is conceivable that additional OCT4 guides those complexes to pluripotency-related targets. This allows more specific reprogramming of target genes relevant to pluripotency, which subsequently stabilize the regulatory network to support development.

\section{Manipulation of Oct4 at later stages of pre-implantation development}

As mentioned, a positive correlation between Oct4 mRNA level and the developmental potential of morula-stage cloned embryos has been reported by our group (Cavaleri et al., 2008). In addition, cloned morula stage embryos generally have lower levels of Oct4 than their fertilized counterparts (Cavaleri et al., 2008). It is important to mind the fact that fertilized Oct4-1- embryos develop to blastocyst at rates comparable to' Oct $4^{+/+}$embryos, indicating that, at least in the presence of maternal Oct4 transcripts and protein, the Oct4 gene itself is dispensable for progression of fertilized embryos beyond the morula stage (Nichols et al., 1998). Furthermore, the relationship between developmental potential and Oct4 levels at the morula stage reported for cloned embryos was not observed in ICSI-fertilized embryos (Cavaleri et al., 2008). Hence the correlation between Oct4 level in the morula stage and development to blastocyst seems unique to cloned embryos and it could either rest on a cause-effect relationship or be readout of earlier events. To solve this dilemma, we used a doxycycline-inducible Oct4 system to manipulate Oct4 levels at later stages of cloned embryo development. Culture of Oct4inducible transgenic cloned embryos in doxycyline-containing medium at the 4- to 8-cell stage did not lead to a significant increase in developmental rates. However, it should be noted, that while an induction of the transgenic Oct4 mRNA was detected, the total amount of Oct4 message increased only slightly, indicating a possible negative regulatory feedback. On the other hand, the difference in Oct4 message between low and high Oct4GFP expressing embryos - predicting low and high developmental success, respectively - is only about $10 \%$, while cloned embryos express only about 60 to $70 \%$ Oct4 levels compared to ICSI counterparts (Cavaleri et al., 2008). Thus, even a minor increase in total Oct4 should have led to a significant increase in development rates. Such increase was not observed, suggesting that Oct4 was not the cause for the increased developmental success of cloned morulae with higher level of Oct4. These results are in agreement with the prevalent view that reprogramming takes place primarily during initial cell cycles. However it is not possible to exclude that the transgenic Oct4 product was somehow less effective. Another possibility is that only embryos with low level of Oct4 at the very beginning could benefit from externally supplied Oct4, whereby no positive effect on developmental potential could be observed when inducing Oct4 at the 4- to 8-cell stage. As it is not possible to determine the amount of Oct4 present in the wild-type oocyte without destroying the cell, or to knockdown Oct4 early on without interfering with later developmental stages as well (siRNA or morpholino), such a relationship cannot be objectively put to the test.

In conclusion, we showed that cloned embryos benefit from additional amounts of Oct4 supplied to the ooplast prior to SCNT. This suggests that Oct4 may play a role in reprogramming by the oocyte, similar to what has been shown in other reprogramming platforms. Furthermore we clarified that the previously reported positive correlation between Oct4 levels at the morula stage and further development of cloned embryos is not due to a cause-effect relationship at the stage considered, but rather reflects superior reprogramming during the first cell divisions. Finally, we demonstrated that manipulation of Oct4 by a triple micromanipulation approach in a nuclear transfer experiment is feasible. This protocol can be used to uncover basic biological principles governing reprogramming, while being unsuited to improve cloning efficiency since the additional micromanipulation step necessary to deliver mRNA reduces the total number of oocytes that can be manipulated in one cloning session. This method is therefore a powerful tool to investigate the role of other putative reprogramming factors like Smarca4 (Egli et al., 2010; Singhal et al., 2010) or activation induced cytidine deaminase (Aid) (Bhutani et al., 2010), to further increase our understanding of the reprogramming machinery of the oocyte.

\section{Materials and Methods}

\section{Molecular cloning}

The coding sequence of mouse Oct 4 was amplified by PCR from the cDNA clone IRAKp961K04111Q2 (ImaGenes) and cloned into pcDNA3.1-FLAG using EcoRI and Xhol. The primers used for PCR were:

5' CGGAATTCGCTGGACACCTGGCTTCA 3' and

5' CGCTCGAGTCAGTTTGAATGCATGGG 3', containing a stop codon (in bold). pcDNA3.1-FLAG was generated from pcDNA3.1 (Invitrogen) using the megaprimer PCR method to insert a FLAG tag-coding cassette upstream of the multi-cloning site. The insertion and sequence of Oct4 in pcDNA3.1-FLAG was verified by Sanger sequencing. The plasmid was transfected into HEK293T cells using Lipofectamine Reagent (Invitrogen \#18324-012) and correct expression of FLAG-OCT4 protein was verified by Western blotting using M2 anti-Flag antibody (Sigma \#F1804).

\section{In vitro transcription}

The pcDNA3.1-FLAG-Oct4 plasmid was linearized using Xhol, then gel purified and subsequently treated with Proteinase $\mathrm{K}$ (Novagen $\# 71049-3)$ in the presence of $0.5 \%$ SDS. After phenol/chloroform purification, $1 \mu \mathrm{g}$ of template DNA was reverse transcribed using the T7 mMessage mMachine kit (Ambion \#AM1344) to yield capped mRNA. The mRNA was Poly $(A)$-tailed using the Poly $(A)$-tailing kit (Ambion \#AM1350) and subsequently purified using the RNeasy mini kit (Qiagen \#74106) according to manufactures instructions. The quality and concentration of the capped and tailed FLAG-Oct4 mRNA was assessed using the Agilent Bioanalyzer 2100 in connection with the Agilent RNA 6000 pico kit (Agilent \#6067-1513). For the production of /uc mRNA, the luciferase encoding control DNA provided in the RiboMAX Large Scale 
RNA Production System (Promega \#TB166) was used. mRNA was transfected into mouse embryonic fibroblast using the TransIT-mRNA Transfection Kit (Mirus \#MIR-2251) and translation of FLAG-Oct4 mRNA was verified by Western blotting using M2 anti-Flag antibody (Sigma \#F1804). Translation of luc mRNA was verified using the Luciferase Assay System (Promega \#E1501).

\section{Mice}

Six- to eight week-old B6C3F1 (C57BI/6J x C3H/HeN) mice were used as oocyte and cumulus cell donors. tetOct 4 mice expressing Oct4 under control of a doxycycline-inducible promoter (strain name B6;129-

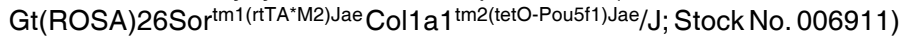
and OG2 mice harboring GFP under the control of the Oct4 promoter (strain Name B6;CBA-Tg(Pou5f1-EGFP)2Mnn/J; Stock No. 004654) are available at Jackson Laboratories. Mice were primed with $10 \mathrm{I}$. .U. each pregnant mare serum gonadotropin (PMSG) and human chorionic gonadotropin (hCG) injected intraperitoneally 48 hours apart. Mice were sacrificed by cervical dislocation 15 hours after hCG and superovulated oocytes were collected from oviducts. Mice were maintained and used for experiments according to institutional guidelines.

\section{Injection of mRNA into zygotes}

Zygotes were retrieved after mating of superovulated B6C3F1 females with CD1 males. In vitro transcribed mRNA was mixed 1 to 50 with red fluorescent dextran beads (Oyster®-568 fluorescent aminodextran 70 kDa, 1 mg/ml; Luminartis GmbH, Münster, Germany) and injected into zygotes with a piezo-operated microcapillary needle $(7 \mu \mathrm{m}$ inner diameter). In vitro culture of embryos was performed in $\alpha$-MEM medium as previously described (Cavaleri et al., 2008). $\alpha$-MEM medium was purchased (Sigma cat. no. M4526) and supplemented wth BSA (2 mg/ml) and gentamicin sulfate $(50 \mu \mathrm{g} / \mathrm{ml})$. Embryos were cultured in $500 \mu \mathrm{l} \alpha$ MEM in Nunc 4-well plates and developmental stage was evaluated at 24, 48 and 96 hours. Embryos were sampled and gene expression analyses as well as immunohistochemistry were performed (see below).

\section{Somatic cell nuclear transfer (SCNT) and intracytoplasmic sperm injection (ICSI)}

SCNT was performed as previously described (Balbach et al., 2007). Briefly, superovulated oocytes were deprived of the chromosomal spindle by gentle suction in a piezo-operated microcapillary needle $(10 \mu \mathrm{m}$ inner diameter, back-loaded with mercury) in the presence of cytochalasin B $(1 \mu \mathrm{g} / \mathrm{ml})$. For the evaluation of the effects of Oct4 increase on early reprogramming events, enucleated ooplasts were injected with $1 \mathrm{ng} / \mu \mathrm{l}$ FLAG-Oct4, or luc mRNA as control. The ooplasts were transplated with single cumulus cell nuclei by injection with a piezo-operated microcapillary needle ( $7 \mu \mathrm{m}$ inner diameter, back-loaded with mercury) in the presence of polyvinylpyrrolidone $40 \mathrm{kDA}(1 \%)$. The nucleus-transplanted ooplasts were parthenogenetically activated in $\mathrm{Ca}$-free $\alpha$-MEM supplemented with $10 \mathrm{mM} \mathrm{SrCl}$ and $5 \mu \mathrm{g} / \mathrm{ml}$ cytochalasin B.

For ICSI, caudae epididymis were removed from mature tetOct4 mice, placed on the bottom of a vial and overlaid with $500 \mu \mathrm{l}$ Whittingham's medium to allow for swim-up of sperm. After 45-60 minutes, the upper $100 \mu \mathrm{l}$ volume of the sperm suspension was gently removed and made into a drop under oil. Sperm and medium were incubated at $37^{\circ} \mathrm{C}$ throughout these steps. After 3 hours, the sperm suspension was spun down gently and resuspended in $10 \mu \mathrm{l} \mathrm{HCZB}$ containing $1 \%$ polyvinylpyrrolidone. Single sperm heads were separated from their tails by a piezo pulse and injected into metaphase II oocytes retrieved from OG2 mice using the same needle as described for SCNT.

All micromanipulations were conducted in HCZB medium (containing $5.56 \mathrm{mM}$ glucose) under Nomarski optics at $30^{\circ} \mathrm{C}$ room temperature. Recovery from micromanipulation was allowed in $\alpha$ MEM medium (ooplasts) or in $\alpha$-MEM and HCZB medium mixed 1:1 (nucleus-transplanted ooplasts and ICSI oocytes) for 1 hour. Developmental progression of SCNT- and ICSI-derived embryos was assessed at 24, 48 and 96 hours and gene expression analyses, as well as immunhistochemistry, were conducted (see below).

\section{qRT-PCR analyses}

For qRT-PCR analyses total RNA was extracted from embryos using the ZR-RNA MicroPrep kit (Zymo Research \#R1060) with an on-column DNA digestion using the RNAse Free DNAse Set (Quiagen \#79254). Complementary DNA synthesis was performed using the High Capacity cDNA Reverse Transcription Kit (Invitrogen \#4368813) according to manufacturers instruction. Transcript levels were determined using the ABI PRISM Sequence Detection System 7900 (Applied BioSystems) in

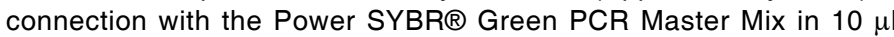
reaction volumes in triplicates. Run conditions were as follows: $50{ }^{\circ} \mathrm{C}$ for 2 minutes, $95^{\circ} \mathrm{C}$ for 10 minutes, $95^{\circ} \mathrm{C}$ for 10 seconds and $60^{\circ} \mathrm{C}$ for 1 minute for a total of 40 cycles; dissociation step: $95^{\circ} \mathrm{C}$ for 15 seconds, $60{ }^{\circ} \mathrm{C}$ for 15 seconds and $95^{\circ} \mathrm{C}$ for 15 seconds. Correct amplification was verified by examining melting curves of PCR products. $C t$-values were obtained with SDS 2.2 (Applied Biosystems), with a threshold of 0.2 and baseline 3 to 15 . Expression levels of different transcripts were normalized to the housekeeping gene Gapdh within the log-linear phase of the amplification curve using the $\Delta \Delta$ Ct method.

Primers for the quantification of the following genes are indicated:

FLAG-Oct4

5' TGGACTACAAGGACGATGATG 3' and

5' GGTGAGAAGGCGAAGTCTGA 3',

Total Oct4

5' AGTATGAGGCTACAGGGACACCTTTC 3' and

5' GGACTGAGTAGAGTGTGGTGAAGTGG-3'

Endogenous Oct4

5' TGTTCCCGTCACTGCTCTGG 3' and

5' TTGCCTTGGCTCACAGCATC 3'.

For experiments with tetOct4 background, primers for the quantification of the following were as indicated:

Transgenic Oct4

5' ATCCACGCTGTTTTGACCTC 3' and

5' CGAAGTCTGAAGCCAGGTGT 3' (Maherali et al., 2007)

Endogenous Oct4

5' GGGTGGGATGGGGAAAGAAG 3' and

5' AGCTATCTACTGTGTGTCCCAGTCTTTATTT 3'.

Primers for Gapdh, used as an internal control, were:

5' CAATGTGTCCGTCGTGGAT 3' and

5' TGCCTGCTTCACCACCTTCT 3'.

\section{Immunohistochemistry, confocal imaging and image analyses}

Immunhistochemistry for Nanog, Oct4 and Cdx2 of blastocyst stage embryos was performed as previously described (Cavaleri et al., 2008). Briefly, embryos were fixed (paraformaldehyde 4\%), permeabilized (Triton $0.1 \%$ in PBS), blocked (BSA $2 \%$, glycine $2 \%$, donkey serum $5 \%$ and Tween $0.1 \%$ in PBS) and incubated in working dilutions of antibodies. As primary antibodies anti-Nanog rabbit igG (Cosmo Bio \#REC-RCAB0002P-F), anti-Oct4 goat IgG (Santa Cruz \#sc 8628) and anti-Cdx2 mouse IgG (Emergo Europe \#CDX2-88) were used. As secondary antibodies anti-rabbit, -goat, -mouse IgG donkey IgG, conjugated with Alexa Fluor 488 (\#A-21206), 568 (\#A-11057) and 647 (\#A-31571), respectively, were applied (all Invitrogen). Imaging was performed with an UltraVIEW RS3 spinning disk confocal imaging system (PerkinElmer LAS) on an inverted microscope (TE2000U, Nikon) using a $60 x$ water immersion lens (N.A. 1.2). The light source was a 3-line (488 nm, $568 \mathrm{~nm}, 647 \mathrm{~nm}$ ) Argon/Krypton laser (Melles Griot). Images were imported into ImageJ (Abramoff et al., 2004) for image analysis.

For quantification of Oct4-promoter driven GFP in Oct4 induced and un-induced cloned embryos, individual morula stage embryos were placed into microdrops of $\alpha$-MEM or $\alpha$-MEM supplemented with $1.25 \mu \mathrm{g} / \mathrm{ml}$ doxycylin on thin-bottom plastic dishes (Lumox, Greiner Bio One 
\#96077333) covered with mineral oil, and confocal images were taken. GFP intensities were quantified with ImageJ. For each morula, the confocal sections were combined (maximal projection) and analyzed with ImageJ. Mean intensities were measured by drawing a region of interest using the polygon tool including only pixels belonging to the embryo; image background was subtracted.

\section{Statistical Analyses}

Statistical analyses were performed using the $R$ program package $(R$ Development Core Team 2009).

\section{Acknowledgements}

We thank David Obridge, Ludger Recker and Christin Schmitz for animal care and experimental support. We also thank the Max-PlanckSociety and Prof. Hans R. Schöler for providing the scientific infrastructure. This work was supported by the special priority program 1356 "Pluripotency and Cellular Reprogramming" of the Deutscheforschungsgemeinschaft (DFG).

\section{References}

BALBACH, S.T., ESTEVES, T.C., BRINK, T., GENTILE, L., MCLAUGHLIN, K.J., ADJAYE, J.A. and BOIANI, M. (2010). Governing cell lineage formation in cloned mouse embryos. Dev Biol 343: 71-83.

BALBACH, S.T., JAUCH, A., BÖHM-STEUER, B., CAVALERI, F.M., HAN, Y.M. and BOIANI, M. (2007). Chromosome stability differs in cloned mouse embryos and derivative ES cells. Dev Biol 308: 309-321.

BHUTANI, N., BRADY, J.J., DAMIAN, M., SACCO, A., CORBEL, S.Y. and BLAU, H.M. (2010). Reprogramming towards pluripotency requires AID-dependent DNA demethylation. Nature 463: 1042-1047.

BOIANI, M., ECKARDT, S., SCHÖLER, H.R. and MCLAUGHLIN, K.J. (2002). Oct4 distribution and level in mouse clones: consequences for pluripotency. Genes Dev 16: 1209-1219.

BOYER, L.A., MATHUR, D. and JAENISCH, R. (2006). Molecular control of pluripotency. Curr Opin Genet Dev 16: 455-462.

BULTMAN, S., GEBUHR, T., YEE, D., LA MANTIA, C., NICHOLSON, J., GILLIAM, A., RANDAZZO, F., METZGER, D., CHAMBON, P., CRABTREE, G. and MAGNUSON, T. (2000). A Brg1 null mutation in the mouse reveals functional differences among mammalian SWI/SNF complexes. Mol Cell 6: 1287-1295.

CAVALERI, F.M., BALBACH, S.T., GENTILE, L., JAUCH, A., BÖHM-STEUER, B., HAN, Y.M., SCHÖLER, H.R. and BOIANI, M. (2008). Subsets of cloned mouse embryos and their non-random relationship to development and nuclear reprogramming. Mech Dev 125: 153-166.

EGLI, D. and EGGAN, K. (2010). Recipient cell nuclear factors are required for reprogramming by nuclear transfer. Development 137: 1953-1963.

FENG, B., NG, J.H., HENG, J.C. and NG, H.H. (2009). Molecules that promote or enhance reprogramming of somatic cells to induced pluripotent stem cells. Cell Stem Cell 3: 301-312.

FOYGEL, K., CHOI, B., JUN, S., LEONG, D.E., LEE, A., WONG, C.C., ZUO, E., ECKART, M., REIJO PERA, R.A., WONG, W.H. and YAO, M.W. (2008). A novel and critical role for Oct4 as a regulator of the maternal-embryonic transition. PLoS One 3: e4109.

HAN, D.W., DO, J.T., GENTILE, L., STEHLING, M., LEE, H.T. and SCHÖLER, H.R. (2008). Pluripotential reprogramming of the somatic genome in hybrid cells occurs with the first cell cycle. Stem Cells 2: 445-454.

HANNA, J., SAHA, K., PANDO, B., VAN ZON, J., LENGNER, C.J., CREYGHTON, M.P., VAN OUDENAARDEN, A. and JAENISCH, R. (2009). Direct cell reprogramming is a stochastic process amenable to acceleration. Nature 462: 595601.

HENG, J.C., FENG, B., HAN, J., JIANG, J., KRAUS, P., NG, J.H., ORLOV, Y.L., HUSS, M., YANG, L., LUFKIN, T., LIM, B. and NG, H.H. (2010). The nuclear receptor $\mathrm{Nr} 5 \mathrm{a} 2$ can replace Oct 4 in the reprogramming of murine somatic cells to pluripotent cells. Cell Stem Cell 6: 167-174.

HOCHEDLINGER, K. and PLATH, K. (2009). Epigenetic reprogramming and induced pluripotency. Development 136: 509-523.

HOCHEDLINGER, K., YAMADA, Y., BEARD, C. and JAENISCH, R. (2005). Ectopic expression of Oct-4 blocks progenitor-cell differentiation and causes dysplasia in epithelial tissues. Cell 6: 465-477.

KIM, J.B., GREBER, B., ARAÚZO-BRAVO, M.J., MEYER, J., PARK, K.I., ZAEHRES, H. and SCHÖLER, H.R. (2009). Direct reprogramming of human neural stem cells by OCT4. Nature 461: 649-654.

KUROSAKA, S., ECKARDT, S. and MCLAUGHLIN, K.J. (2004) Pluripotent lineage definition in bovine embryos by Oct4 transcript localization. Biol Reprod 71: 1578-1582.

LIU, S., LIU, H., PAN, Y., TANG, S., XIONG, J., HUI, N., WANG, S., QI, Z. and LI, L. (2004). Human embryonic germ cells isolation from early stages of postimplantation embryos. Cell Tissue Res 318: 525-531.

MAHERALI, N., SRIDHARAN, R., XIE, W., UTIKAL, J., EMINLI, S., ARNOLD, K., STADTFELD, M., YACHECHKO, R., TCHIEU, J., JAENISCH, R., PLATH, K. and HOCHEDLINGER, K. (2007). Directly reprogrammed fibroblasts show global epigenetic remodeling and widespread tissue contribution. Cell Stem Cell 1: 55-77.

MONTI, M. and REDI, C. (2009). Oogenesis specific genes (Nobox, Oct4, Bmp15, Gdf9, Oogenesin1 and Oogenesin2) are differentially expressed during natura and gonadotropin-induced mouse follicular development. Mol Reprod Dev 76: 994-1003.

NICHOLS, J., ZEVNIK, B., ANASTASSIADIS, K., NIWA, H., KLEWE-NEBENIUS, D., CHAMBERS, I., SCHÖLER, H. and SMITH, A. (1998). Formation of pluripotent stem cells in the mammalian embryo depends on the POU transcription factor Oct4. Cell 95: 379-391.

NIWA, H., MIYAZAKI, J. and SMITH, A.G. (2000). Quantitative expression of Oct$3 / 4$ defines differentiation, dedifferentiation or self-renewal of ES cells. Nat Genet 24: 372-376.

PAPAPETROU, E.P., TOMISHIMA, M.J., CHAMBERS, S.M., MICA, Y., REED, E., MENON, J., TABAR, V., MO, Q., STUDER, L. and SADELAIN, M. (2009). Stoichiometric and temporal requirements of Oct4, Sox2, Klf4, and c-Myc expression for efficient human iPSC induction and differentiation. Proc Nat Acad Sci 106: 12759-12764.

PARDO, M., LANG, B., YU, L., PROSSER, H., BRADLEY, A., BABU, M.M. and CHOUDHARY, J. (2010). An expanded Oct4 interaction network: implications for stem cell biology, development, and disease. Cell Stem Cell 6: 382-395

PESCE, M., GROSS, M.K. and SCHÖLER, H.R. (1998). In line with our ancestors: Oct-4 and the mammalian germ. Bioessays 20: 722-732.

R Development Core Team (2009). R: A language and environment for statistical computing. R Foundation for Statistical Computing, Vienna, Austria.

SINGHAL, N., GRAUMANN, J., WU, G., ARAÚZO-BRAVO, M.J., HAN, D.W. GREBER, B., GENTILE, L., MANN, M. and SCHÖLER, H.R. (2010). ChromatinRemodeling components of the BAF complex facilitate reprogramming. Cell 141:943-955.

TAKAHASHI, K. and YAMANAKA, S. (2006). Induction of pluripotent stem cells from mouse embryonic and adult fibroblast cultures by defined factors. Cell 126 : 663-676.

TANG, S., WANG, Y., ZHANG, D., GAO, Y., MA, Y., YIN, B., SUN, J., LIU, J. and ZHANG, Y. (2009). Reprogramming donor cells with oocyte extracts improves in vitro development of nuclear transfer embryos. Anim Reprod Sci 115: 1-9.

TSUJI, I., MITANI, T., MITSUHASHI, A., WATANABE, Y., HOSOI, Y. and HOSHIAI, $H$. (2006). Inhibition of Oct4 expression in mouse preimplantation embryos using morpholino antisense oligonucleotides. Tohoku J Exp Med208: 333-342.

VAN DEN BERG, D.L., SNOEK, T., MULLIN, N.P., YATES, A., BEZSTAROSTI, K., DEMMERS, J., CHAMBERS, I. and POOT, R.A. (2010). An Oct4-centered protein interaction network in embryonic stem cells. Cell Stem Cell 6: 369-381.

ZHAO, X.Y., LI, W., LV, Z., LIU, L., TONG, M., HAI, T., HAO, J., GUO, C.L., MA, Q.W., WANG, L., ZENG, F. and ZHOU, Q. (2009). iPS cells produce viable mice through tetraploid complementation. Nature 461: 86-90. 


\section{Further Related Reading, published previously in the Int. J. Dev. Biol.}

See our recent Special Issue Placenta edited by Joan S. Hunt and Kent L. Thornburg at: http://www.ijdb.ehu.es/web/contents.php?vol=54\&issue=2-3

The effect of superovulation on the contributions of individual blastomeres from 2-cell stage CF1 mouse embryos to the blastocyst. Katayama, M. and Roberts, R.M.

Int J Dev Biol (2010) 54: 675-681.

Factors engaged in reactivation of DNA replication in the nuclei of growing mouse oocytes introduced into the cytoplasm of parthenogenetic one-cell embryos.

Borsuk, E. and Czolowska, R.

Int J Dev Biol (2010) 54: 21-31.

Reprogramming of melanoma cells by embryonic microenvironments.

Díez-Torre, A., Andrade, R., Eguizábal, C., López, E., Arluzea, J., Silió, M. and Aréchaga, J.

Int J Dev Biol (2009) 53: 1563 - 1568.

Pluripotency and differentiation in embryos and stem cells - Pavia, 17-18 January 2008.

Adjaye, J.A., Byskov, A.G., Cibelli, J.B, de Maria, R., Minger, S., Sampaolesi, M., Testa, G., Verfaillie, C., Zernicka-Goetz, M., Schöler, H., Boiani, M., Crosetto, N. and Redi, C.A.

Int J Dev Biol (2008) 52: 801-809.

Mammalian oocyte activation: lessons from the sperm and implications for nuclear transfer.

Alberio, R., Zakhartchenko, V., Motlik, J. and Wolf, E.

Int J Dev Biol (2001) 45: 797-809.

Epigenetic reprogramming of the genome from the germ line to the embryo and back again.

Arney, K.L., Erhardt, S., Drewell, R.A. and Surani, M.A.

Int J Dev Biol (2001) 45: 533-540.

Changes in embryonic 8-cell nuclei transferred by means of cell fusion to mouse eggs.

Czolowska, R., Szöllösi, D. and Szöllösi, M.S.

Int J Dev Biol (1992) 36: 543-553.

5 yr ISI Impact Factor $(2009)=3.253$

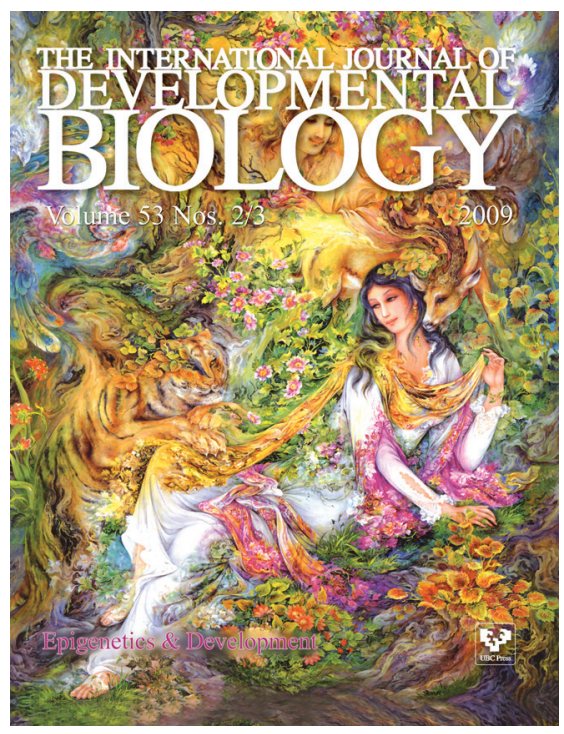

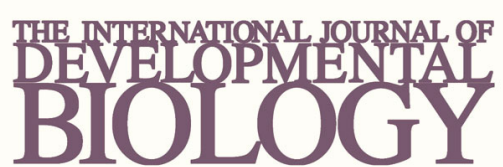

Volume 54 Nos. $6 / 7$
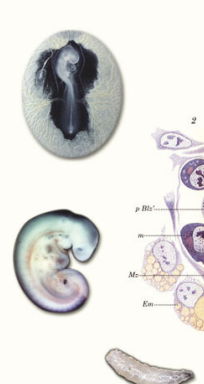

Developmental Hematopoiesis
Special Issue
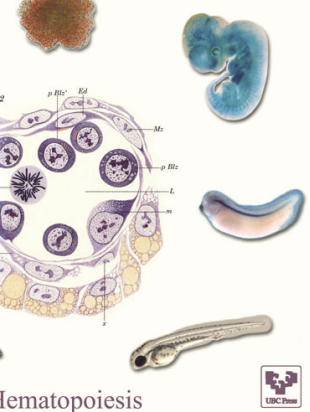

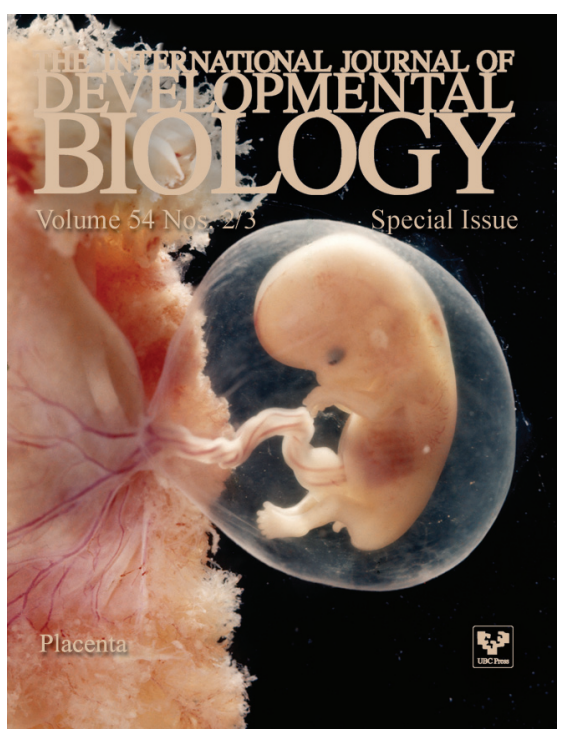

